\title{
Can a Small Nodal Metastasis Reliably Alter Intraoperative Risk/Benefit Decision Making in Breast Cancer Surgery?
}

\author{
Lisa K. Jacobs, MD, and Charles Balch, MD \\ Johns Hopkins University, 600 N Wolfe Street, Osler 624, Baltimore, MD 21287, USA
}

Surgeons treating breast cancer patients make repeated decisions as they select from an array of complex management options. The end point of any recommendation to our patients is, do the potential benefits of surgery outweigh the potential risks? Whether to perform an axillary lymphadenectomy and whether to go ahead with autologous tissuebased immediate breast reconstructive surgery immediately after total mastectomy is determined by intraoperative evaluation of the sentinel node or nodes removed from the axilla.

Regarding the decisions surrounding the staging and treatment of axillary lymph nodes, the elements of decision making on the benefits side include: (1) the goals of surgery (diagnosis of metastases, regional disease control, and increasing survival rates), (2) staging information on the natural history of disease that affects survival outcomes (e.g., number of nodal metastases, extracapsular invasion), and (3) improved cosmetic and rehabilitation benefits of immediate breast reconstruction using autologous flaps. The elements of decision making on the risk side include: (1) the morbidity of a level I and II lymphadenectomy (e.g., wound complications, lymphedema, numbness), (2) patient comorbidity risk factors (e.g., obesity, older age), (3) risk of not performing a lymphadenectomy, which may increase the future risk of regional recurrence, and (4) the risk of poor outcome after reconstruction followed by irradiation to the reconstructed breast.

Received July 11, 2007; accepted July 13, 2007; published online: September 19, 2007.

Address correspondence and reprint requests to: Lisa K. Jacobs, MD; E-mail: ljacob14@jhmi.edu

Published by Springer Science+Business Media, LLC $\odot 2007$ The Society of Surgical Oncology, Inc.
One central component of this decision-making is getting complete and reliable information on the status of nodal metastases, which in turn influences intraoperative decisions on whether to perform a more complete lymphadenectomy and decisions on the type of immediate breast reconstructive surgery. The staging information regarding nodal status will also influence postoperative decisions regarding the type and intensity of adjuvant systemic therapy and the deployment of adjuvant radiation therapy to the remaining axillary/supraclavicular lymph nodes and the chest wall.

Some tenets of lymph nodes metastases are being reexamined as sentinel lymph node (SLN) technology is increasingly being used in the staging of and treatment planning for breast cancer and melanoma. Samoilova et al. ${ }^{1}$ ask a provocative question: is it necessary to perform a more complete axillary node dissection in patients with small micrometastases in the SLN? Alternatively, could the treatment safely be limited to the sentinel node excision or a limited dissection of level I lymph nodes? And what is the lower threshold of metastatic cells in a SLN for which we should stage patients as $\mathrm{N}+$ instead of $\mathrm{N}(\mathrm{mic})$, or dismiss the finding as isolated tumor cells of no clinical significance?

One of the important questions addressed in this study is whether any factors would predict an incidence of four or more metastatic nodes for which adjuvant radiation therapy would be indicated and for which extensive reconstructive breast surgery might have to be deferred until this is completed. The authors performed a retrospective review of 467 patients who underwent SLN biopsy, 126 (27\%) of whom were found to have metastases in the SLN. They then correlated clinicopathological variables 
with the incidence of finding additional metastases in non-SLNs after an axillary lymphadenectomy. The authors performed a careful evaluation of the SLN by studying $2-\mathrm{mm}$ intervals by examination of both frozen sections and hematoxylin and eosin-stained samples. In this study, any evidence of tumor cells within the sentinel node on hematoxylin and eosin stain was recorded as a disease-positive node. Patients with a disease-positive SLN underwent completion lymphadenectomy. Approximately 20\% of the SLN-positive patients (and 5\% of all patients) had more than three metastatic nodes (N2-3). The measured dimensions of the nodal metastases were the prognostic variable that most reliably separated N1a from N2-3 patients. All patients with sentinel node deposits $<5 \mathrm{~mm}$ had three or fewer metastatic nodes, and $91 \%$ had a single nodal metastases. As the authors state in this retrospective study, this information could be valuable in making intraoperative decisions as to whether it would be safe to perform a limited node dissection, or even no further surgical excision other than excision of the SLNs.

The authors state that a complete axillary lymphadenectomy "is associated with significant morbidity: lymphedema is reported in $13 \%$ to $50 \%$, sensory loss in $31 \%$, and overall arm edema in $38 \%$ of patients." Understanding morbidity risks when making intraoperative decisions is important information in assessing the risk-benefit ratio of lymphadenectomy. But we believe these estimates overstate the morbidity risks in the current era of breast surgery. The risk-benefit analysis must also include the risk of the SLN procedure and the future risk to the patient if an axillary recurrence does occur. The best risk analysis of the SLN procedure comes from the American College of the Surgeons Oncology Group Z0010 data, which showed a 6.9\% incidence of arm swelling (change from baseline arm circumference of $>2 \mathrm{~cm}$ ) at 6 months after surgery - a risk greater than was anticipated. The sentinel node procedure also carries a risk of axillary seroma of $7.1 \%$, hematoma $1.4 \%$, wound infection $1 \%$, axillary paresthesias $8.6 \%$, and decreased range of motion $3.8 \%$. These risks are reported with 6 months of follow-up, but the long-term effects are currently unknown. ${ }^{2}$

How solid is the evidence that a more complete lymphadenectomy fulfills the goals of staging and treatment? How solid is the evidence that selective lymphadenectomy of the nodes surrounding the SLN fulfills the goals of staging and treatment? If limited lymph node dissection is completed and the disease recurs in the axilla, what are the treatment options, and what is the morbidity associated with these options? We do not know the recurrence rates when a limited dissection is completed. What is the morbidity of these limited dissections? What is the nerve injury rate, and what is the lymphedema rate?

What is the evidence of increased risk after a more complete lymphadenectomy? Publication of the ALMANAC trial demonstrates an increased risk of axillary dissection over SLN biopsy. ${ }^{3}$ However, the clinical significance of the absolute risks documented in that study are not known. Investigation into the use of more limited nodal dissection (levels I/II) must include the impact on morbidity and risk of relapse in an era of micrometastases?

What is the evidence that irradiation after breast reconstructive surgery causes tissue distortions and poor outcomes? What is the influence of specific findings on frozen-section examination of the sentinel node in patients undergoing total mastectomy and the number of metastatic nodes on intraoperative decisions to proceed with axillary dissection, autologous tissue reconstruction, and postoperative irradiation? Should plans continue for immediate breast reconstruction, and should it influence the type of reconstruction used?

The authors have concluded that sentinel node patients with tumor deposits of $<5 \mathrm{~mm}$ will only have a 5\% incidence of non-SLN metastases and that there is a $91 \%$ probability of a single node involvement. From this retrospective data, they concluded that such patients may be considered for a limited node dissection-limited to sentinel nodes only and/or a selective axillary dissection of only the nodes immediately adjacent to the SLN. We believe this is a spurious conclusion because the denominator of patients to be compared is really the $27 \%$ of patients who had a metastatic sentinel node, not the entire group of patients undergoing a SLN procedure, including those who were SLN negative. The more representative incidence on more than three metastatic nodes (N2-3) is 19\% (24 of 126 SLN-positive patients). This higher incidence mitigates the argument that one could forego a more complete lymphadenectomy because of the risk that patients might be understaged, and therefore undertreated, if they did not have a level I and II lymphadenectomy. Nevertheless, it does provide an important hypothesis to test in prospective clinical trials on the natural history of small micrometastases. 
If there is no therapeutic benefit for complete lymphadenectomy in SLN-positive patients, then the primary value of the procedure is staging. If the results from this retrospective study were confirmed by a prospective study, then this group of patients with tiny metastases might be able to join those who are sentinel node negative and be spared the additional treatment and associated morbidity of a more complete lymphadenectomy. On the other hand, we have an obligation to prove that we can provide more conservative treatment safely, so this study cannot be used to alter what is today considered the standard of care.

\section{REFERENCES}

1. Samoilova E, Brill Y, Davis J, et al. Size of sentinel node tumor deposits and extent of axillary lymph node involvement: which breast cancer patients may benefit from less aggressive axillary dissections?. Ann Surg Oncol 2007; 14:2223-9.

2. Wilke LG, McCall LM, Posther KE, et al. Surgical complications associated with sentinel lymph node biopsy: results from a prospective international cooperative group trial. Ann Surg Oncol 2006; 13:491-500.

3. Fleissig A, Fallowfield LJ, Langridge CI, et al. Post-operative arm morbidity and quality of life. Results of the ALMANAC randomised trial comparing sentinel node biopsy with standard axillary treatment in the management of patients with early breast cancer. Breast Cancer Res Treat 2006; 95:279-93. 